\title{
Sex Differences in Cardiovascular Mortality in Diabetics and Nondiabetic Subjects: A Population-Based Study (Italy)
}

\author{
Paola Ballotari, ${ }^{1,2}$ Sofia Chiatamone Ranieri, ${ }^{3}$ Ferdinando Luberto, ${ }^{1,2}$ Stefania Caroli, ${ }^{1,2}$ \\ Marina Greci, ${ }^{4}$ Paolo Giorgi Rossi, ${ }^{1,2}$ and Valeria Manicardi ${ }^{5}$ \\ ${ }^{1}$ Servizio Interaziendale di Epidemiologia, Azienda Unità Sanitaria Locale, Via Amendola 2, 42122 Reggio Emilia, Italy \\ ${ }^{2}$ IRCCS Arcispedale Santa Maria Nuova, Viale Umberto I 50, 42123 Reggio Emilia, Italy \\ ${ }^{3}$ Laboratorio Analisi Chimico Cliniche ed Endocrinologia, IRCCS Arcispedale Santa Maria Nuova, Viale Risorgimento 80, 42123 \\ Reggio Emilia, Italy \\ ${ }^{4}$ Dipartimento Cure Primarie, Azienda Unità Sanitaria Locale, Via Amendola 2, 42122 Reggio Emilia, Italy \\ ${ }^{5}$ Dipartimento di Medicina Interna, Ospedale di Montecchio, Azienda Unità Sanitaria Locale, Via Barilla 16, 42027 Montecchio, Italy
}

Correspondence should be addressed to Sofia Chiatamone Ranieri; sofia.chiatamoneranieri@gmail.com

Received 3 July 2014; Revised 3 October 2014; Accepted 5 October 2014

Academic Editor: Giuseppina T. Russo

Copyright (C) 2015 Paola Ballotari et al. This is an open access article distributed under the Creative Commons Attribution License, which permits unrestricted use, distribution, and reproduction in any medium, provided the original work is properly cited.

The objective of this study is to assess the impact of diabetes on cardiovascular mortality, focusing on sex differences. The inhabitants of Reggio Emilia province on December 31, 2009, aged 20-84 were followed up for three years for mortality. The exposure was determined using Reggio Emilia diabetes register. The age-adjusted death rates were estimated as well as the incidence rate ratios using Poisson regression model. Interaction terms for diabetes and sex were tested by the Wald test. People with diabetes had an excess of mortality, compared with nondiabetic subjects (all cause: IRR $=1.68$; 95\%CI 1.60-1.78; CVD: IRR $=1.61$; 95\%CI 1.47-1.76; AMI: IRR $=1.59 ; 95 \%$ CI 1.27-1.99; renal causes: IRR $=1.71 ; 95 \%$ CI 1.22-2.38). The impact of diabetes is greater in females than males for all causes $(P=0.0321)$ and for CVD, IMA, and renal causes. Further studies are needed to investigate whether the difference in cardiovascular risk profile or in the quality of care delivered justifies the higher excess of mortality in females with diabetes compared to males.

\section{Introduction}

Diabetes is now one of the most common noncommunicable diseases globally. The International Diabetes Federation (IDF) most recent estimates indicate that $8.3 \%$ of adults382 million people-have diabetes. Further, the number of people with the disease is expected to rise beyond 592 million in less than 25 years. Yet with 175 million cases currently undiagnosed, a vast number of people who are unaware that they have diabetes are progressing towards complications [1]. In Italy, the overall prevalence in 2011 was about 5\%; that is, $1,383,000$ men and 1,556,000 women have diabetes [2].

Diabetes and its complications are major causes of early death in most countries. In Europe, one in 10 deaths in adults can be attributed to diabetes, that is 619,000 in 2013 [1]. Cardiovascular disease (CVD), the first cause of death in many industrialized countries, is responsible for a large part of the excess mortality observed among people with diabetes [3]. Indeed, individuals with diabetes have an increased risk of all-cause mortality and morbidity related to CVD compared with individuals without diabetes [3-9].

Nevertheless, the effect of diabetes on CVD seems to be different for males and females [10-17]. In fact, despite the fact that in many industrialized countries women have lower mortality rates than men, when we look at people with diabetes, the advantage for women is reduced or even absent $[18,19]$. Estimates of CVD mortality in men with diabetes have varied from 1 to 3 times the rate in men free of the disease, whereas estimates in women with diabetes have ranged from 2 to 5 times the rate in women without diabetes [20-23]. The variation in relative risk estimates of cardiovascular disease makes it difficult to evaluate the strength of diabetes as a risk factor for either sex. 
The objective of this study is therefore to assess the impact of diabetes on cardiovascular mortality, focusing on sex differences.

\section{Methods}

2.1. Setting and Study Population. This study is a retrospective cohort including the inhabitants of Reggio Emilia province (northern Italy) on December 31, 2009, aged 20-84.

To identify people with diabetes (i.e., exposed group) we used the Reggio Emilia diabetes register (accessed on May 21, 2014). The methods applied to develop our disease register have been described elsewhere [24]. In brief, the register was created by deterministic linkage of six routinely collected data sources through a definite algorithm able to ascertain cases and to distinguish type of diabetes and model of care. The sources are hospital discharge, drug dispensation, HbAlc values from biochemistry laboratory, disease-specific exemption, diabetes outpatient clinics, and mortality databases. Women with gestational diabetes or women receiving treatment for polycystic ovarian syndrome were excluded.

2.2. Follow-Up, Outcome, and Covariates. Cohort was followed up for three years (2010-2012). Vital status (alive or dead) information was retrieved from civil register. The subjects who emigrated were treated as censored at the time of emigration.

The outcome of interest was mortality attributable to all causes (ICD-10 A00-T98), cardiovascular disease (CVD) (ICD-110 I00-I99), acute myocardial infarction (AMI) (ICD10 I21-I23), diabetes (ICD-10 E10-E14), and renal diseases (ICD-10 N00-N39). The causes of death were ascertained using Reggio Emilia mortality register, which contains all resident deaths by year of death, with cause of death coded using International Classification of Diseases, tenth revision (ICD-10). Sex and age were considered covariates in the analysis. As a proxy of disease severity, the subjects with diabetes were classified based on treatment: diet only, oral antidiabetic drugs, or insulin. Subjects who were prescribed both insulin and oral antidiabetic drugs were assigned to "insulin treatment" [25].

2.3. Statistical Methods. Characteristics of the study population are presented as median and proportions and stratified by sex and diabetes status. Person-time at risk was calculated from January 1, 2010, to date of death or date of emigration or December 31, 2012.

We calculated proportional mortality by age and diabetes status for principal groups of cause of death.

Then we estimated age-adjusted death rates (AADR) per 100000 with $95 \%$ confidence intervals (95\% CI), by sex and diabetes status using Italian population on December 31, 2009, as reference for standardization [26]. At the same time, we calculated incidence rate ratios (IRR) and 95\% confidence intervals (95\% CI) using multivariate Poisson regression model. The individuals without diabetes were used as the reference group, the age as continuous variable, and the sex as covariate. Interaction terms for diabetes and sex were tested by the Wald test.

Further, we estimated incidence rate ratios (IRR) and 95\% confidence intervals (95\% CI) and risk difference (per 100000) within age category, for all causes, CVD, and AMI and renal causes, and we graphed the age-specific death rates stratified by sex and diabetes status.

Analyses were performed using the STATA statistical package, version 11.0.

2.4. Ethical Approval. This is an observational study and data were collected retrospectively. The Local Health Authority of Reggio Emilia was responsible for collecting and processing these sets of data. The study was commissioned by the Local Health Authority. The Reggio Emilia diabetes registry was approved by provincial Ethic Committee in July 2014. According to Italian privacy law, no patient or relative's consent is required for large retrospective population-based studies.

\section{Results}

The study cohort consisted of 407,161 subjects (Table 1), 23,438 of whom were diabetic patients (i.e., exposed group) (5.8\% of the population): 13074 males and 10364 females (prevalence $6.5 \%$ and $5.0 \%$, resp.). Subjects without diabetes were younger and there was a higher percentage of foreigners. The percentage of lost to follow-up because of move was very low in both groups.

Over the three-year study period, 9,208 (2.3\%) individuals died; the proportion of deaths was higher in people with diabetes than the unexposed population $(8.7 \%$ and $1.9 \%$, resp.). The risk was greater in males than females in both groups.

Finally, among people with diabetes, there were no differences by sex in terms of type of treatment $(P=0.120)$.

The distribution of causes was similar for the two populations (Table 2), with the exception of death for endocrine, nutritional, and metabolic causes (which includes diabetes) (E00-E90), where the percentage was $10.3 \%$ for males and $11.4 \%$ for females with diabetes, compared to $0.7 \%$ and $0.9 \%$ for males and females without diabetes, respectively. The pattern of mortality by sex was similar in the two subgroups, except for the digestive and renal causes. In females, the proportion of deaths for CVD was 33.2\%, with a slight difference between diabetics and nondiabetics subjects (34.4\% and $32.9 \%$ resp.); in males the percentage of deaths for CVD causes was lower (28.5\%) and similar in the two groups.

Diabetic subjects showed an increased risk of all-cause mortality compared to nondiabetics of dying for all causes (Table 3). The excess of risk was found in all categories of causes analyzed in our study.

The analysis by sex indicated that the excess of risk was more evident in diabetic females than diabetic males compared to their nondiabetic counterparts (IRR 1.77; 95\% CI 1.64-1.92; IRR 1.63; 95\% CI 1.52-1.73, resp.). The effect modification of sex on the association between diabetes and 
TABLE 1: Characteristics of the study cohort by diabetes status and sex.

\begin{tabular}{|c|c|c|c|c|c|c|}
\hline \multirow{2}{*}{ Characteristics } & \multicolumn{2}{|c|}{ No diabetes } & \multicolumn{2}{|c|}{ Diabetes } & \multicolumn{2}{|c|}{ Total } \\
\hline & Males & Females & Males & Females & Males & Females \\
\hline Population $20-84$ years & 187886 & 195837 & 13074 & 10364 & 200960 & 206201 \\
\hline Foreigners ${ }^{*}: N(\%)$ & $25749(13.7)$ & $28104(14.3)$ & $950(7.3)$ & $862(8.3)$ & $26699(13.3)$ & $28966(14.0)$ \\
\hline Age (years): median (IQR) & $44(34-59)$ & $47(35-62)$ & $66(57-74)$ & $69(60-76)$ & $46(35-61)$ & $48(36-63)$ \\
\hline Dead: $N(\%)$ & $4090(2.2)$ & $3090(1.6)$ & $1240(9.5)$ & $788(7.6)$ & $5330(2.6)$ & $3878(1.9)$ \\
\hline Emigrated: N (\%) & $417(0.2)$ & $319(0.2)$ & $16(0.1)$ & $12(0.1)$ & $433(0.2)$ & $331(0.2)$ \\
\hline Person-years & 558521 & 583162 & 37234 & 29842 & 595755 & 613004 \\
\hline \multicolumn{7}{|l|}{ Diabetes treatment regimen } \\
\hline Diet only & & & $3213(24.6)$ & $2626(25.3)$ & & \\
\hline Oral drugs & & & $6760(51.7)$ & $5219(50.4)$ & & \\
\hline Insulin & & & $3101(23.7)$ & $2519(24.3)$ & & \\
\hline
\end{tabular}

${ }^{*}$ Based on the country of birth.

TABle 2: Proportional mortality by diabetes status and sex.

\begin{tabular}{|c|c|c|c|c|c|c|}
\hline \multirow{2}{*}{ Causes: $N(\%)$} & \multicolumn{2}{|c|}{ No diabetes } & \multicolumn{2}{|c|}{ Diabetes } & \multicolumn{2}{|c|}{ Total } \\
\hline & Males & Females & Males & Females & Males & Females \\
\hline $\begin{array}{l}\text { Infectious and parasitic diseases } \\
\text { (A00-B99) }\end{array}$ & $97(2.4)$ & $72(2.3)$ & $39(3.1)$ & $30(3.8)$ & $136(2.6)$ & $102(2.6)$ \\
\hline Neoplasms (C00-D48) & $1680(41.1)$ & $1176(38.1)$ & $426(34.4)$ & $239(30.3)$ & $2106(39.5)$ & $1415(36.5)$ \\
\hline $\begin{array}{l}\text { Endocrine, nutritional, and metabolic } \\
\text { diseases (E00-E90) }\end{array}$ & $30(0.7)$ & $29(0.9)$ & $128(10.3)$ & $90(11.4)$ & $158(3.0)$ & $119(3.0)$ \\
\hline $\begin{array}{l}\text { Mental and behavioral disorders } \\
\text { (F00-F99) }\end{array}$ & $61(1.5)$ & $87(2.8)$ & $13(1.0)$ & $7(0.9)$ & $74(1.4)$ & $94(2.4)$ \\
\hline $\begin{array}{l}\text { Diseases of the nervous system } \\
\text { (G00-G99) }\end{array}$ & $146(3.6)$ & $132(4.3)$ & $19(1.5)$ & $23(2.9)$ & $165(3.1)$ & $155(4.0)$ \\
\hline $\begin{array}{l}\text { Diseases of the circulatory system } \\
\text { (I00-I99) }\end{array}$ & $1161(28.4)$ & $1017(32.9)$ & $357(28.8)$ & $271(34.4)$ & $1518(28.5)$ & $1288(33.2)$ \\
\hline $\begin{array}{l}\text { Diseases of the respiratory system } \\
\text { (J00-J99) }\end{array}$ & $335(8.2)$ & $205(6.6)$ & $102(8.2)$ & $38(4.8)$ & $437(8.2)$ & $243(6.3)$ \\
\hline $\begin{array}{l}\text { Diseases of the digestive system } \\
\text { (K00-K93) }\end{array}$ & $154(3.8)$ & $130(4.2)$ & $67(5.4)$ & $36(4.6)$ & $221(4.1)$ & $166(4.3)$ \\
\hline Renal causes (N00-N99) & $87(2.1)$ & $59(1.9)$ & $25(2.0)$ & $21(2.7)$ & $112(2.1)$ & $80(2.1)$ \\
\hline $\begin{array}{l}\text { Injury, poisoning, and other certain } \\
\text { consequences of external causes } \\
\text { (S00-T98) }\end{array}$ & $244(6.6)$ & $110(3.6)$ & $44(3.5)$ & $17(2.2)$ & $288(5.4)$ & $127(3.3)$ \\
\hline Unknown & $46(1.1)$ & $22(0.7)$ & $13(1.0)$ & $5(0.6)$ & $59(1.1)$ & $27(0.7)$ \\
\hline Other ${ }^{*}$ & $49(1.2)$ & $51(1.7)$ & $7(0.6)$ & $11(1.4)$ & $56(1.1)$ & $62(1.6)$ \\
\hline Total (A00-T98) & 4090 & 3090 & 1240 & 788 & 5330 & 3878 \\
\hline
\end{tabular}

* Others include cases classified in the following chapters: III, Diseases of the blood and blood-forming organs and certain disorders involving the immune mechanism (D50-D89) $(N=20)$; VII, Diseases of the eye and adnexa $(\mathrm{H} 00-\mathrm{H} 59)(N=1)$; VIII, Diseases of the ear and mastoid process (H60-H95) $(N=1)$; XII, Diseases of the skin and subcutaneous tissue (L00-L99) $(N=14)$; XIII, Diseases of the musculoskeletal system and connective tissue (M00M99) ( $N=34$ ); XVII, Congenital malformations, deformations and chromosomal abnormalities (Q00-Q99) ( $N=11)$; XVIII, Symptoms, signs and abnormal clinical and laboratory findings, not elsewhere classified (R00-R99) $(N=37)$.

death was statistically significant (Wald test for interaction, $P=0.0321)$.

Looking at cardiovascular mortality, we observed a similar pattern: an excess of risk in people with diabetes, found in both sexes, greater in females than males (males: IRR 1.56; 95\% CI 1.38-1.76; females: IRR 1.69; 95\% CI 1.47-1.93; Wald test for interaction, $P=0.1266$ ).

Among the CVD causes, we observed that for AMI the excess mortality for females with diabetes was more pronounced (males: IRR 1.48; 95\% CI 1.10-1.99; females: 1.81; 95\% CI 1.27-2.59; Wald test for interaction, $P=0.1063$ ).

In the group of renal causes of death, the excess of mortality in the diabetic population was again more evident in females than males (males: IRR 1.37; 95\% CI 0.88-2.14; females: 2.37; 95\% CI 1.43-3.91; Wald test for interaction, $P=$ 0.1466). This group of causes includes those related to kidney dysfunctions, such as glomerular diseases, renal tubulointerstitial diseases, acute kidney failure, chronic kidney disease, 


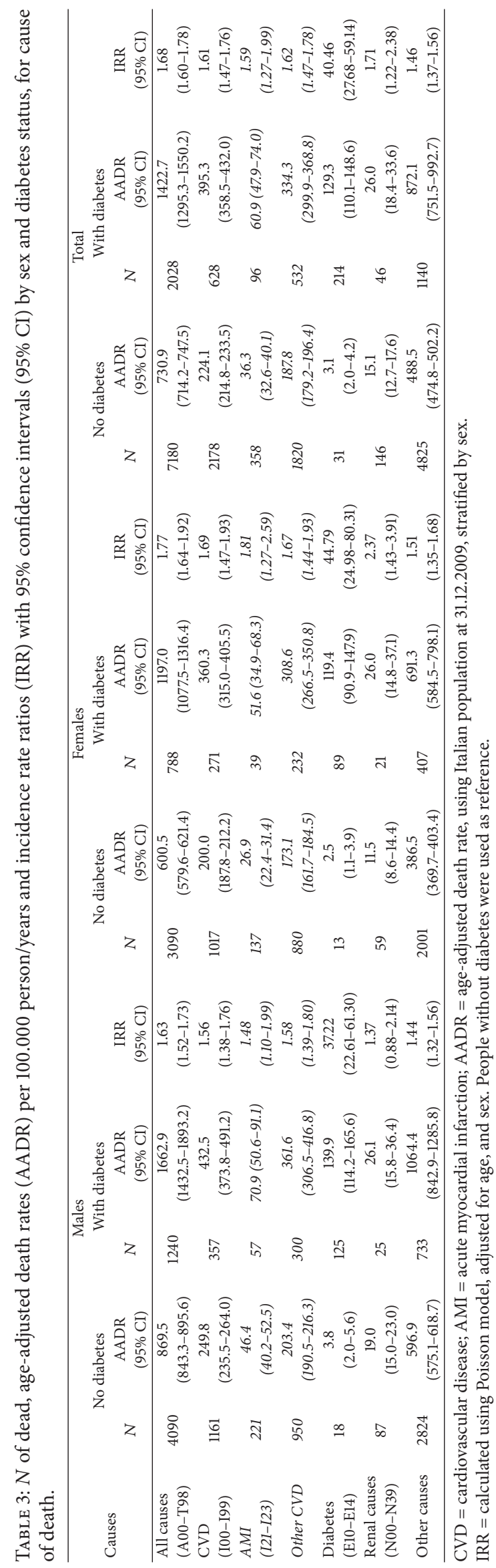




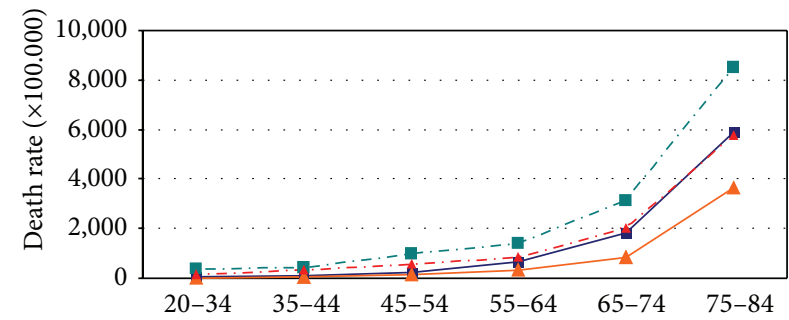

(a) All causes

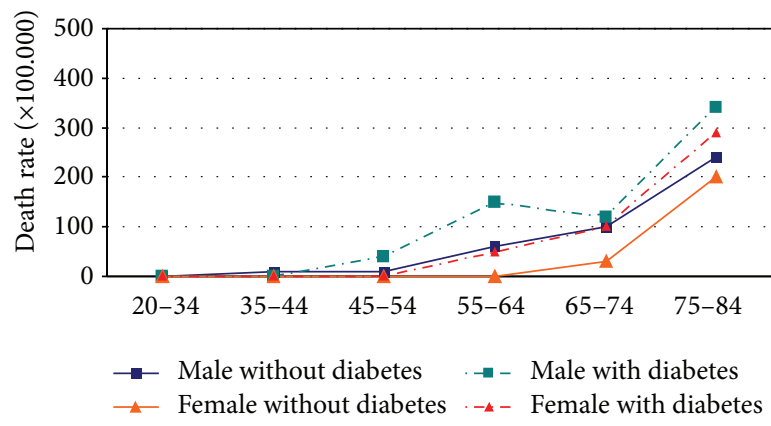

(c) AMI

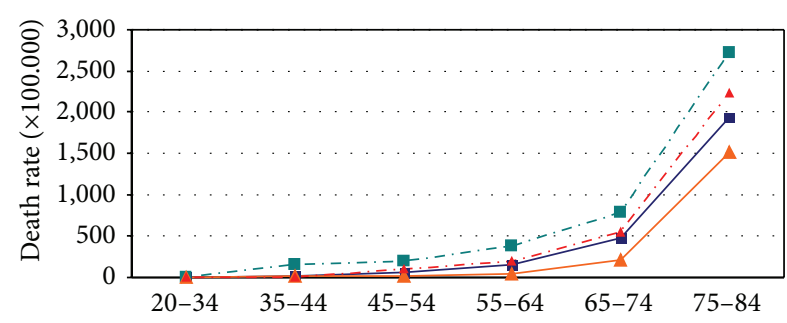

(b) CVD

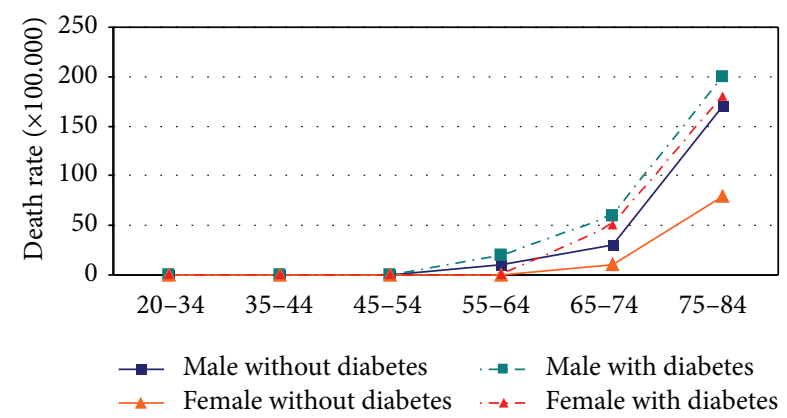

(d) Renal causes

FIgure 1: Age-specific death rates by sex and diabetes status: (a) all cause of death; (b) CVD; (c) AMI; (d) renal causes. The curves with dash indicate diabetic patients and the curve with squares indicate males and the curve with triangles indicate females.

and other disorders of the kidney and urethra. In this group, the deaths caused by renal failures were $70 \%$ of the total in the diabetic population, while the percentage decreased to $59 \%$ in nondiabetic population. In both subgroups, the remaining deaths were almost entirely ascribed to "other diseases of urinary system" block.

Comparing number of deaths by cause among diabetic and nondiabetic individuals, we observed 214 deaths caused by diabetes in the former subgroup and 31 in the latter (this subgroup included people with diabetes diagnosed after 2009), corresponding to a cause-specific age-adjusted death rate of 129.3 and 3.1 per $100000 \mathrm{p} / \mathrm{y}$, respectively. The presence of the diabetes-specific cause makes it difficult to compare the other causes of mortality between the two populations. In fact, this cause of death subtracts cases to other causes and in particular to cardiovascular and renal causes, because often the final cause was attributable to one of these two categories.

Analysis of incidence rate ratios by age class suggests that the impact of diabetes decreases with increasing age (Table 4). The effect can only be observed in all-cause mortality and CVD as a whole, because the absolute AMI and renal causes risk of death are too small in younger ages. Nevertheless, the risk difference increased with age, reaching 26.3 per $1000 \mathrm{p} / \mathrm{y}$ in males aged 75-84 for all causes and 21.1 in females, while in 20-34-year-old class the difference was 1.6 per 1000 p/y in males and 1.3 in females. In case of CVD, the risk difference in the oldest age class reached 7 per $1000 \mathrm{p} / \mathrm{y}$, in both sexes.

Comparison among age-specific death rates by sex and diabetes status (Figure 1) indicated that males with diabetes have the highest rates. However, females with diabetes have higher rates than males without diabetes mainly in the younger age groups, while females without diabetes have very low death rates until the age of 64 .

\section{Discussion}

Our study found an excess of mortality associated with diabetes in both sexes, for all causes and for all groups of causes analyzed. However, the excess in the ratios was limited compared to findings of other studies [9, 27-29]. It must be emphasized that our study was population-based and data on exposure were retrieved from a register built using six different sources, assuring sensitivity and specificity [24]. This study design includes a wider denominator of exposed people compared to studies where the cohort is hospital or treatment based.

Focusing on CVD causes, the risk of death for diabetics is $61 \%$ higher than that for nondiabetics subjects (95\% CI: $1.47-$ 1.76), with no differences between the two subcategories, AMI and "other CVD causes".

Considering all causes of death, our study found evidence of greater impact of diabetes on females than males, despite the severity of disease seeming to be similar in the two groups, in agreement with a recent population-based retrospective cohort study [18]. When we analyzed CVD, IMA, and renal causes, the different effect of diabetes by sex was also present, although the power of the study does not permit ruling out the possibility that the difference was due to random fluctuations.

The reason why diabetes determines a greater excess of all-cause mortality in females than males is not completely understood, especially for CVD causes [30]. 


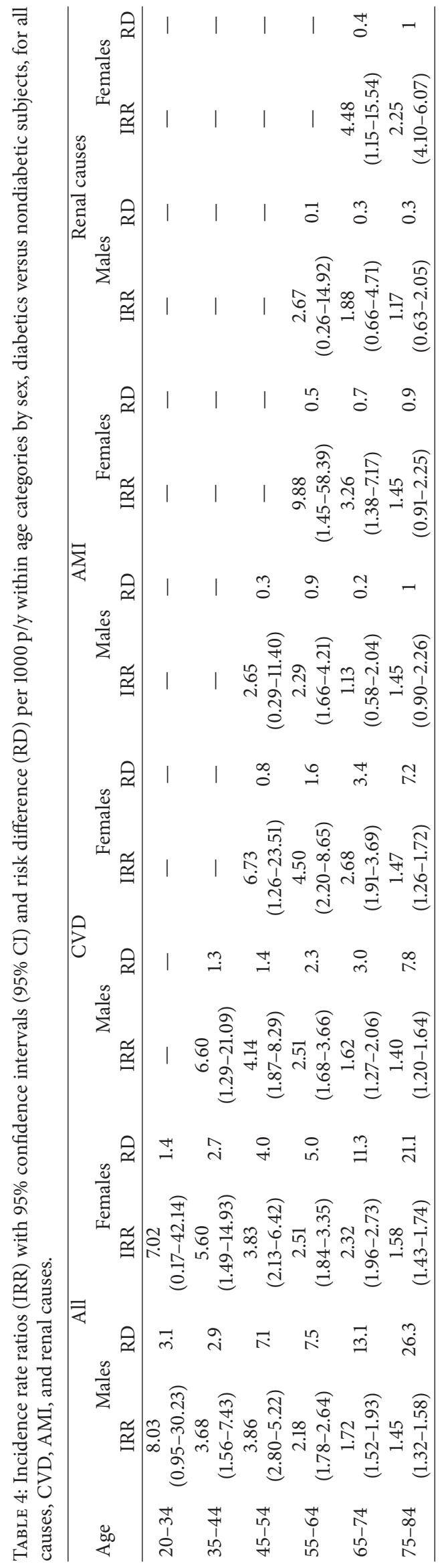


One explanation is that type-2 diabetes mellitus (T2DM) may reduce the advantage of females in the prevalence of cardiovascular disease by fading the vascular protective effects given by estrogens [21, 31-33]. Many authors have suggested that the CVD risk factors have a stronger impact on females than males $[14,15,32-34]$. Compared to males, females with diabetes have a worse cardiovascular profile, which could explain their higher cardiovascular mortality, mainly at age $<60$. Females with diabetes have higher prevalent abdominal obesity [34, 35], increasing the risk of hypertension $[19,36]$, a worse lipid profile, since the onset of diabetes (low levels of HDL cholesterol [HDL-C], small particle size of LDL cholesterol [LDL-C], and high levels of triglycerides) [35, 37-39], and a more marked endothelial dysfunction than males with diabetes [40-45], a greater degree of fibrinolysis/thrombosis compared to males $[46,47]$, and also an increased prevalence of hypoglycemic events compared to that of male diabetic patients [48]. These phenomena might explain the increased incidence of cardiovascular events and mortality among female patients [49].

Besides innate differences in sex physiology, disparities between sexes in the treatment of major cardiovascular risk factors also exist $[35,50,51]$. These can be attributed to an underestimation of patient risk and a less aggressive approach (i.e., prescription of lower doses) and poorer compliance of females [52-55]. Nevertheless, two Italian studies did not find any relevant differences between females and males in terms of the quality of diabetes care $[35,56]$. In one of these recent large cross-sectional studies, women were less likely to reach the recommended targets despite receiving the same treatment for lipid control and hypertension and they were more likely to be overtreated with insulin. Women still showed a lower likelihood of being monitored for diabetes complications, particularly foot and eye complications. As for intermediate outcomes, the proportion of individuals reaching the targets of $\mathrm{HbAlc}$, LDL cholesterol, and BMI values was systematically lower for women than men. The only result that went in the opposite direction was that, among diabetic patients with high LDL cholesterol, a higher proportion of women were not treated with lipid-lowering therapy [35].

In our study we also investigated mortality caused by renal diseases and in particular codes N00-N39, that is, glomerular diseases, renal tubulointerstitial diseases, acute kidney failure and chronic kidney disease, urolithiasis, other disorders of kidney and urethra, and other diseases of the urinary system, given the close interconnection between renal and cardiovascular disease.

For this group as well, we found risk excess in diabetic population and the excess was stronger in females than males. This excess in females is closely linked to CVD mortality and could partially explain its increase [57-59]. There are few sets of data on the role of gender on microvascular complications and increasing mortality related to them [60]. While females in the general population have less renal disease, this advantage is less evident in diabetic nephropathy than nondiabetic kidney diseases [61-63]. The contribution of sex to diabetic renal disease is still unclear. Although some studies indicate that females progress at a faster rate [64], others studies indicate the opposite [65-67]. Some studies suggest that male sex remains a risk factor for the development of micro- and macroalbuminuria as well as the progression of an established diabetic nephropathy [68]. However, the prevalence of a reduced glomerular filtration rate estimated in females was higher than that in males [69]. This nonalbuminuric renal impairment phenotype is associated with higher incidence of CVD, particularly in the coronary district [70].

The differences in therapy effectiveness in females as well as the existence of different disease pathways in the kidney and cardiovascular disease have led some authors to suggest the need to develop gender-specific therapeutic strategies to prevent renal dysfunction and reduce associated morbidity and mortality in females [71].

Nevertheless, it is important to note that even if the incidence rate ratios declined with age, the risk difference increased in the older groups, where the number of deaths is much higher. In other words, in a hypothetical population free of diabetes disease, in age class 75-84 years, 26 deaths for every 1,000 males and 21 deaths for 1,000 females still alive would be avoided, while the savings would be 1.6 for 1,000 males and 1.3 for 1,000 females in the age class $20-34$ years.

The particularly high excess of risk in younger ages is mainly due to low mortality in nondiabetic group, and the phenomenon is more pronounced in females. Our results agree with other studies $[69,70]$.

\section{Strengths and Limitations}

This is a population-based cohort study using data from a province-wide diabetes register for exposure identification and from mortality register for case detection, thereby reducing misclassification bias. Moreover, while there have been several studies on all causes and CVD mortality among people with diabetes, this is one of the few studies exploring the effect of diabetes on renal causes mortality. Finally, our study focused on the greater impact of diabetes on female mortality, exploring possible hypotheses for this phenomenon.

However, this study considered only age as confounder; other possible confounders, such as socioeconomic characteristics, behavioral risk factors (i.e., BMI, smoking), and clinical information other than treatment, such as duration of disease and micro- and macrovascular diabetes complications, were not considered.

Finally, the presence of diabetes as cause of death makes it difficult to compare the cause-specific mortality between the population with and without diabetes, in particular for CVD and renal diseases.

\section{Conclusions}

Diabetes determines a $68 \%$ excess in mortality rate. The relative risk for diabetic patients versus nondiabetic population is particularly relevant in young and middle-aged subjects, where diabetes status contributes to occurrence of deaths that are unexpected in nondiabetic population. Furthermore, diabetes has a greater impact on females than males, reducing 
the advantage of females in all-cause mortality as well as CVD, in particular AMI, and renal mortality observed in the population without diabetes.

Further studies are needed to determine whether the difference in cardiovascular risk profile or the quality of care delivered justifies the higher excess of mortality in females with diabetes than males.

\section{Conflict of Interests}

The authors declare that there is no conflict of interests regarding the publication of this paper.

\section{Acknowledgment}

The study has been partially founded by the INMP (Istituto Nazionale per la promozione della salute delle popolazioni Migranti ed il contrasto della Povertà) with the grant "La rete degli Studi Longitudinali metropolitani per la valutazione dei profili di assistenza della popolazione immigrate.

\section{References}

[1] International Diabetes Federation, IDF Diabetes Atlas, International Diabetes Federation, Brussels, Belgium, 6th edition, 2013.

[2] ISTAT, "Indagine Multiscopo 'Aspetti della vita quotidiana," in Anno 2011, ISTAT, Rome, Italy, 2012.

[3] WHO, "World Health Organisation Study Group on Diabetes Mellitus," Tech. Rep. 727, WHO, Geneva, Switzerland, 1985.

[4] R. J. Jarrett, "Diabetes, hyperglycaemia and arterial disease," in Complications of Diabetes, H. Keen and R. J. Jarrett, Eds., pp. 179-203, Edward Arnold, London, UK, 2nd edition, 1982.

[5] G. L. Booth, M. K. Kapral, K. Fung, and J. V. Tu, "Relation between age and cardiovascular disease in men and women with diabetes compared with non-diabetic people: a population-based retrospective cohort study," The Lancet, vol. 368, no. 9529, pp. 29-36, 2006.

[6] S. R. Preis, S.-J. Hwang, S. Coady et al., "Trends in all-cause and cardiovascular disease mortality among women and men with and without diabetes mellitus in the framingham heart study, 1950 to 2005," Circulation, vol. 119, no. 13, pp. 1728-1735, 2009.

[7] T. Almdal, H. Scharling, J. S. Jensen, and H. Vestergaard, “The independent effect of type 2 diabetes mellitus on ischemic heart disease, stroke, and death: a population-based study of 13,000 men and women with 20 years of follow-up," Archives of Internal Medicine, vol. 164, no. 13, pp. 1422-1426, 2004.

[8] K. N. Barnett, S. A. Ogston, M. E. T. McMurdo, A. D. Morris, and J. M. M. Evans, "A 12-year follow-up study of all-cause and cardiovascular mortality among 10532 people newly diagnosed with Type 2 diabetes in Tayside, Scotland," Diabetic Medicine, vol. 27, no. 10, pp. 1124-1129, 2010.

[9] N. Sarwar, P. Gao, S. R. Seshasai, R. Gobin, S. Kaptoge, and The Emerging Risk Factors Collaboration, "Diabetes mellitus, fasting blood glucose concentration, and risk of vascular disease: a collaborative meta-analysis of 102 prospective studies," The Lancet, vol. 375, pp. 2215-2222, 2010.

[10] S. Vilbergsson, S. Vilbergsson, G. Sigurdsson, G. Sigurdsson, H. Sigvaldason, and N. Sigfusson, "Coronary heart disease mortality amongst non-insulin-dependent diabetic subjects in Iceland: the independent effect of diabetes. The Reykjavik Study 17-year follow up," Journal of Internal Medicine, vol. 244, no. 4, pp. 309-316, 1998.

[11] W. J. Butler, L. D. Ostrander Jr., W. J. Carman, and D. E. Lamphiear, "Mortality from coronary heart disease in the tecumseh study. Long-term effect of diabetes mellitus, glucose tolerance and other risk factors," The American Journal of Epidemiology, vol. 121, no. 4, pp. 541-547, 1985.

[12] J. C. Kleinman, R. P. Donahue, M. I. Harris, F. F. Finucane, J. H. Madans, and D. B. Brock, "Mortality among diabetics in a national sample," The American Journal of Epidemiology, vol. 128, no. 2, pp. 389-401, 1988.

[13] G.-M. Lin, Y.-H. Li, C.-L. Lin, J.-H. Wang, and C.-L. Han, "Gender differences in the impact of diabetes on mortality in patients with established coronary artery disease: a report from the eastern Taiwan integrated health care delivery system of Coronary Heart Disease (ET-CHD) registry, 1997-2006," Journal of Cardiology, vol. 61, no. 6, pp. 393-398, 2013.

[14] A. M. Kanaya, D. Grady, and E. Barrett-Connor, "Explaining the sex difference in coronary heart disease mortality among patients with type 2 diabetes mellitus: a meta-analysis," Archives of Internal Medicine, vol. 162, no. 15, pp. 1737-1745, 2002.

[15] B. V. Howard, L. D. Cowan, O. Go, T. K. Welty, D. C. Robbins, and E. T. Lee, "Adverse effects of diabetes on multiple cardiovascular disease risk factors in women: the strong heart study," Diabetes Care, vol. 21, no. 8, pp. 1258-1265, 1998.

[16] A. Becker, G. Bos, F. de Vegt et al., "Cardiovascular events in type 2 diabetes: comparison with nondiabetic individuals without and with prior cardiovascular disease: 10-Year followup of the Hoorn study," European Heart Journal, vol. 24, no. 15, pp. 1406-1413, 2003.

[17] A. Juutilainen, S. Kortelainen, S. Lehto, T. Rönnemaa, K. Pyörälä, and M. Laakso, "Gender difference in the impact of type 2 diabetes on coronary heart disease risk," Diabetes Care, vol. 27, no. 12, pp. 2898-2904, 2004.

[18] M. M. Roche and P. P. Wang, "Sex differences in all-cause and cardiovascular mortality, hospitalization for individuals with and without diabetes, and patients with diabetes diagnosed early and late," Diabetes Care, vol. 36, no. 9, pp. 2582-2590, 2013.

[19] E. Barrett-Connor, E.-G. V. Giardina, A. K. Gitt, U. Gudat, H. O. Steinberg, and D. Tschoepe, "Women and heart disease: the role of diabetes and hyperglycemia," Archives of Internal Medicine, vol. 164, no. 9, pp. 934-942, 2004.

[20] W. B. Kannel, "The Framingham Study: Historical insight on the impact of cardiovascular risk factors in men versus women," Journal of Gender-Specific Medicine, vol. 5, no. 2, pp. 27-37, 2002.

[21] E. L. Barrett-Connor, B. A. Cohn, D. L. Wingard, and S. L. Edelstein, "Why is diabetes mellitus a stronger risk factor for fatal ischemic heart disease in women than in men? The Rancho Bernardo Study," Journal of the American Medical Association, vol. 265, no. 5, pp. 627-631, 1991.

[22] W. L. Lee, A. M. Cheung, D. Cape, and B. Zinman, "Impact of diabetes on coronary artery disease in women and men: a metaanalysis of prospective studies," Diabetes Care, vol. 23, no. 7, pp. 962-968, 2000.

[23] R. Huxley, F. Barzi, and M. Woodward, "Adverse effects of diabetes on multiple cardiovascular disease risk factors in women: the strong heart study," British Medical Journal, vol. 332, no. 7533, pp. 73-76, 2006.

[24] P. Ballotari, S. Chiatamone Ranieri, M. Vicentini et al., "Building a population-based diabetes register: an Italian experience," Diabetes Research and Clinical Practice, vol. 103, no. 1, pp. 7987, 2014. 
[25] R. Gnavi, R. Picariello, L. la Karaghiosoff, G. Costa, and C. Giorda, "Determinants of quality in diabetes care process: the population-based Torino study," Diabetes Care, vol. 32, no. 11, pp. 1986-1992, 2009.

[26] ISTAT, Resident population on December 31st, 2012, http:// www.demo.istat.it/bil2012/index.html.

[27] N. Sarwar, P. Gao, and S. R. Seshasai, "Diabetes mellitus, fasting blood glucose concentration, and risk of vascular disease: a collaborative meta-analysis of 102 prospective studies," The Lancet, vol. 375, no. 9733, pp. 2215-2222, 2010.

[28] T. K. Schramm, G. H. Gislason, L. Køber et al., "Diabetes patients requiring glucose-lowering therapy and nondiabetics with a prior myocardial infarction carry the same cardiovascular risk: a population study of 3.3 million people," Circulation, vol. 117, no. 15, pp. 1945-1954, 2008.

[29] K. S. Taylor, C. J. Heneghan, A. J. Farmer et al., "All-cause and cardiovascular mortality in middle-aged people with type 2 diabetes compared with people without diabetes in a large U.K. primary care database," Diabetes Care, vol. 36, no. 8, pp. 23662371, 2013.

[30] E. W. Gregg, Q. Gu, Y. J. Cheng, K. M. V. Narayan, and C. C. Cowie, "Mortality trends in men and women with diabetes, 1971 to 2000," Annals of Internal Medicine, vol. 147, no. 3, pp. 149-155, 2007.

[31] H. H. Newnham and J. Silberberg, "Coronary heart disease. Women's hearts are hard to break," The Lancet, vol. 349, pp. S13S16, 1997.

[32] C. E. Walden, R. H. Knopp, P. W. Wahl, K. W. Beach, and E. Strandness Jr., "Sex differences in the effect of diabetes mellitus on lipoprotein triglyceride and cholesterol concentrations," The New England Journal of Medicine, vol. 311, no. 15, pp. 953-959, 1984.

[33] R. D. Siegel, A. Cupples, E. J. Schaefer, and P. W. F. Wilson, "Lipoproteins, apolipoproteins, and low-density lipoprotein size among diabetics in the Framingham offspring study," Metabolism, vol. 45, no. 10, pp. 1267-1272, 1996.

[34] G. Penno, A. Solini, E. Bonora et al., "Gender differences in cardiovascular disease risk factors, treatments and complications in patients with type 2 diabetes: the RIACE Italian multicentre study," Journal of Internal Medicine, vol. 274, no. 2, pp. 176-191, 2013.

[35] M. C. Rossi, M. R. Cristofaro, S. Gentile et al., "Sex disparities in the quality of diabetes care: biological and cultural factors may play a different role for different outcomes: a cross-sectional observational study from the amd annals initiative," Diabetes Care, vol. 36, no. 10, pp. 3162-3168, 2013.

[36] M. J. Legato, A. Gelzer, R. Goland et al., "Gender-specific care of the patient with diabetes: review and recommendations," Gender Medicine, vol. 3, no. 2, pp. 131-158, 2006.

[37] J. Dallongevillle, D. de Bacquer, J. Heidrich et al., "Gender differences in the implementation of cardiovascular prevention measures after an acute coronary event," Heart, vol. 96, no. 21, pp. 1744-1749, 2010.

[38] V. G. Vimalananda, D. R. Miller, M. Palnati, C. L. Christiansen, and B. G. Fincke, "Gender disparities in lipid-lowering therapy among veterans with diabetes," Womens Health Issues, vol. 21, no. 4 supplement, pp. S176-S181, 2011.

[39] C. Kim, E. A. Kerr, S. J. Bernstein, and S. L. Krein, "Gender disparities in lipid management: the presence of disparities depends on the quality measure," American Journal of Managed Care, vol. 12, no. 3, pp. 133-136, 2006.
[40] A. Festa, R. D’Agostino Jr., R. P. Tracy, and S. M. Haffner, "Elevated levels of acute-phase proteins and plasminogen activator inhibitor-1 predict the development of type 2 diabetes: the insulin resistance atherosclerosis study, Diabetes, vol. 51, no. 4, pp. 1131-1137, 2002.

[41] L. H. Kuller, R. P. Tracy, J. Shaten, and E. N. Meilahn, "Relation of C-reactive protein and coronary heart disease in the MRFIT nested case-control study," American Journal of Epidemiology, vol. 144, no. 6, pp. 537-547, 1996.

[42] J. B. Meigs, F. B. Hu, N. Rifai, and J. E. Manson, "Biomarkers of endothelial dysfunction and risk of type 2 diabetes mellitus," Journal of the American Medical Association, vol. 291, no. 16, pp. 1978-1986, 2004.

[43] P. M. Ridker, M. Cushman, M. J. Stampfer, R. P. Tracy, and C. H. Hennekens, "Inflammation, aspirin, and the risk of cardiovascular disease in apparently healthy men," The New England Journal of Medicine, vol. 336, no. 14, pp. 973-979, 1997.

[44] M. I. Schmidt, B. B. Duncan, A. R. Sharrett et al., "Markers of inflammation and prediction of diabetes mellitus in adults (Atherosclerosis Risk in Communities study): a cohort study," The Lancet, vol. 353, no. 9165, pp. 1649-1652, 1999.

[45] H. O. Steinberg, G. Paradisi, J. Cronin et al., “Type II diabetes abrogates sex differences in endothelial function in premenopausal women," Circulation, vol. 101, no. 17, pp. 20402046, 2000.

[46] F. Franconi, I. Campesi, S. Occhioni, and G. Tonolo, "Sexgender differences in diabetes vascular complications and treatment," Endocrine, Metabolic and Immune Disorders-Drug Targets, vol. 12, no. 2, pp. 179-196, 2012.

[47] R. P. Donahue, K. Rejman, L. B. Rafalson, J. Dmochowski, S. Stranges, and M. Trevisan, "Sex differences in endothelial function markers before conversion to pre-diabetes: does the clock start ticking earlier among women? The Western New York Study," Diabetes Care, vol. 30, no. 2, pp. 354-359, 2007.

[48] M. E. Miller, D. E. Bonds, H. C. Gerstein et al., "The effects of baseline characteristics, glycaemia treatment approach, and glycated haemoglobin concentration on the risk of severe hypoglycaemia: post hoc epidemiological analysis of the ACCORD study," BMJ, vol. 340, no. 7738, Article ID b5444, p. 138, 2010.

[49] R. R. Kalyani, M. Lazo, P. Ouyang et al., "Sex differences in diabetes and risk of incident coronary artery disease in healthy young and middle-aged adults," Diabetes Care, vol. 37, no. 3, pp. 830-838, 2014

[50] A. Ferrara, C. M. Mangione, C. Kim et al., "Sex disparities in control and treatment of modifiable cardiovascular disease risk factors among patients with diabetes," Diabetes Care, vol. 31, no. 1, pp. 69-74, 2008.

[51] D. J. Wexler, R. W. Grant, J. B. Meigs, D. M. Nathan, and E. Cagliero, "Sex disparities in treatment of cardiac risk factors in patients with type 2 diabetes," Diabetes Care, vol. 28, no. 3, pp. 514-520, 2005.

[52] R. Bugiardini, A. T. Yan, R. T. Yan et al., "Factors influencing underutilization of evidence-based therapies in women," European Heart Journal, vol. 32, no. 11, pp. 1337-1344, 2011.

[53] S. Poon, S. G. Goodman, R. T. Yan et al., "Bridging the gender gap: insights from a contemporary analysis of sexrelated differences in the treatment and outcomes of patients with acute coronary syndromes," American Heart Journal, vol. 163, no. 1, pp. 66-73, 2012.

[54] S. H. Saydah, J. Fradkin, and C. C. Cowie, "Poor control of risk factors for vascular disease among adults with previously 
diagnosed diabetes," The Journal of the American Medical Association, vol. 291, no. 3, pp. 335-342, 2004.

[55] J. L. Jarvie and J. M. Foody, "Recognizing and improving health care disparities in the prevention of cardiovascular disease in women," Current Cardiology Reports, vol. 12, no. 6, pp. 488-496, 2010.

[56] C. Giorda, R. Picariello, E. Nada et al., "The impact of adherence to screening guidelines and of diabetes clinics referral on morbidity and mortality in diabetes," PLoS ONE, vol. 7, no. 4, Article ID e33839, 2012.

[57] J. P. Garg and G. L. Bakris, "Microalbuminuria: marker of vascular dysfunction, risk factor for cardiovascular disease," Vascular Medicine, vol. 7, no. 1, pp. 35-43, 2002.

[58] K. Klausen, K. Borch-Johnsen, B. Feldt-Rasmussen et al., "Very low levels of microalbuminuria are associated with increased risk of coronary heart disease and death independently of renal function, hypertension, and diabetes," Circulation, vol. 110, no. 1, pp. 32-35, 2004.

[59] G. T. Russo, A. Giandalia, E. L. Romeo et al., "Lipid and non-lipid cardiovascular risk factors in postmenopausal type 2 diabetic women with and without coronary heart disease," Journal of Endocrinological Investigation, vol. 37, no. 3, pp. 261268, 2014.

[60] N. A. Roper, R. W. Bilous, W. F. Kelly, N. C. Unwin, and V. M. Connolly, "Cause-specific mortality in a population with diabetes: South tees diabetes mortality study," Diabetes Care, vol. 25, no. 1, pp. 43-48, 2002.

[61] S. L. Seliger, C. Davis, and C. Stehman-Breen, "Gender and the progression of renal disease," Current Opinion in Nephrology and Hypertension, vol. 10, no. 2, pp. 219-225, 2001.

[62] S. R. Silbiger and J. Neugarten, "The role of gender in the progression of renal disease," Advances in Renal Replacement Therapy, vol. 10, no. 1, pp. 3-14, 2003.

[63] C. J. Schultz, T. Konopelska-Bahu, R. N. Dalton et al., "Microalbuminuria prevalence varies with age, sex, and puberty in children with type 1 diabetes followed from diagnosis in a longitudinal study," Diabetes Care, vol. 22, no. 3, pp. 495-502, 1999.

[64] R. Garza, R. Medina, S. Basu, and J. A. Pugh, "Predictors of the rate of renal function decline in non-insulin-dependent diabetes mellitus," American Journal of Nephrology, vol. 17, no. 1, pp. 59-67, 1997.

[65] P. Jacobsen, K. Rossing, L. Tarnow et al., "Progression of diabetic nephropathy in normotensive type 1 diabetic patients," Kidney International, Supplement, vol. 56, no. 71, pp. S101-S105, 1999.

[66] M. I. Harris, K. M. Flegal, C. C. Cowie et al., "Prevalence of diabetes, impaired fasting glucose, and impaired glucose tolerance in U.S. adults: the Third National Health and Nutrition Examination Survey, 1988-1994," Diabetes Care, vol. 21, no. 4, pp. 518-524, 1998.

[67] H. E. Resnick and B. V. Howard, "Diabetes and cardiovascular disease," Annual Review of Medicine, vol. 53, pp. 245-267, 2002.

[68] C. Maric, "Sex, diabetes and the kidney," American Journal of Physiology-Renal Physiology, vol. 296, no. 4, pp. F680-F688, 2009.

[69] A. Ceriello, S. de Cosmo, S. Gentile et al., Le Monografie Degli Annali AMD 2011 Focus Su: Pattern Assistenziali in Relazione al Livello di Funzionalità Renale, 2012.

[70] A. Solini, G. Penno, E. Bonora et al., "Diverging association of reduced glomerular filtration rate and albuminuria with coronary and noncoronary events in patients with type 2 diabetes: The renal insufficiency and cardiovascular events (RIACE) Italian multicenter study," Diabetes Care, vol. 35, no. 1, pp. 143-149, 2012.

[71] M. L. Muiesan, E. Ambrosioni, F. V. Costa et al., "Sex differences in hypertension-related renal and cardiovascular diseases in Italy: the I-DEMAND study," Journal of Hypertension, vol. 30, no. 12, pp. 2378-2386, 2012. 


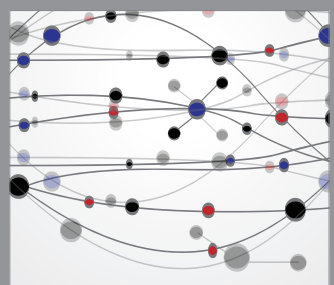

The Scientific World Journal
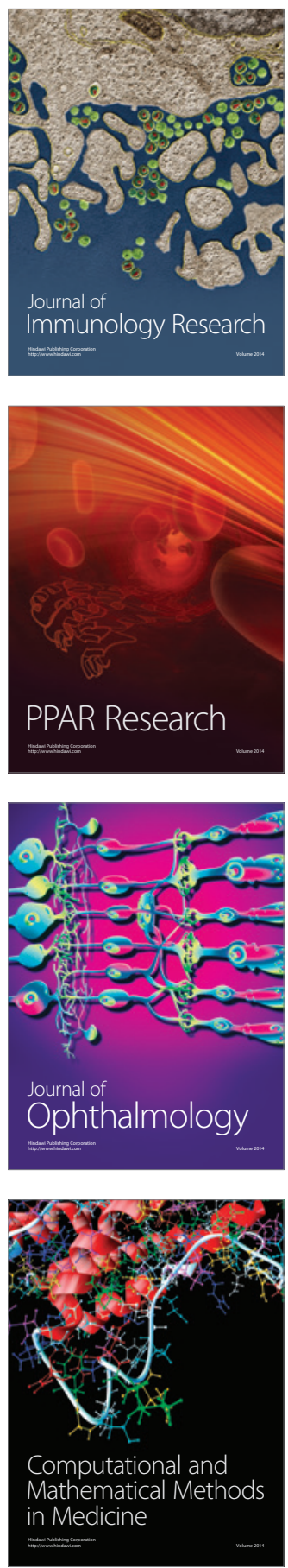

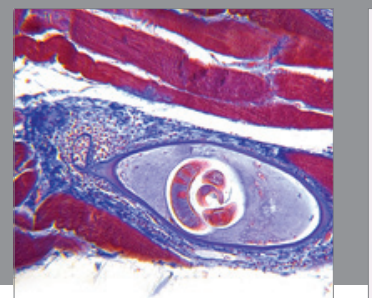

Gastroenterology

Research and Practice
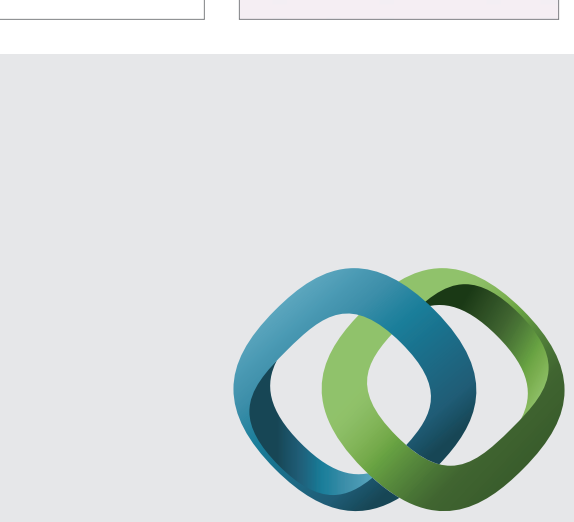

\section{Hindawi}

Submit your manuscripts at

http://www.hindawi.com
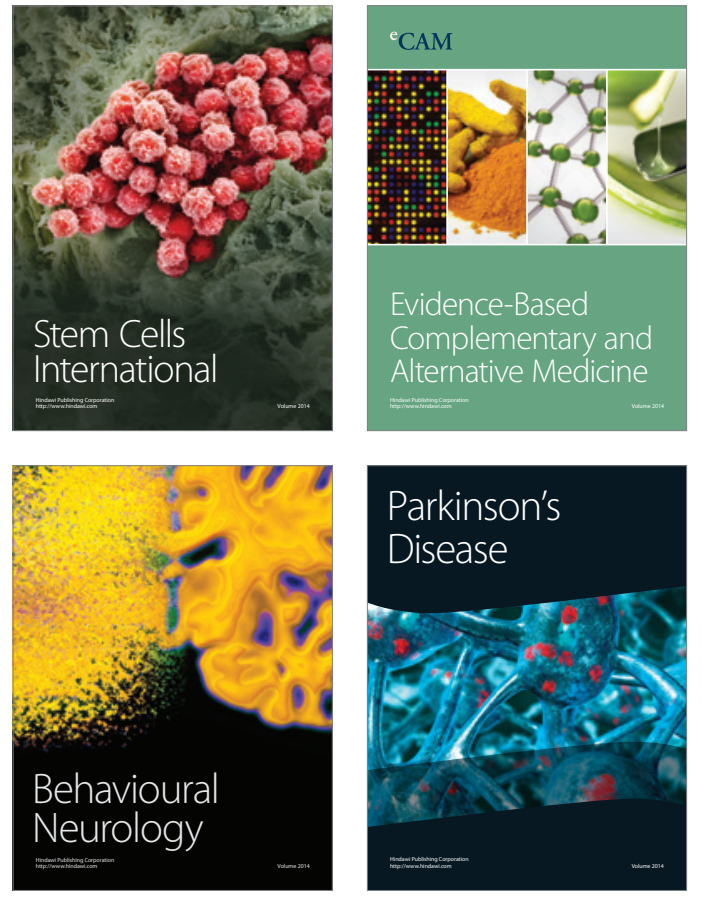
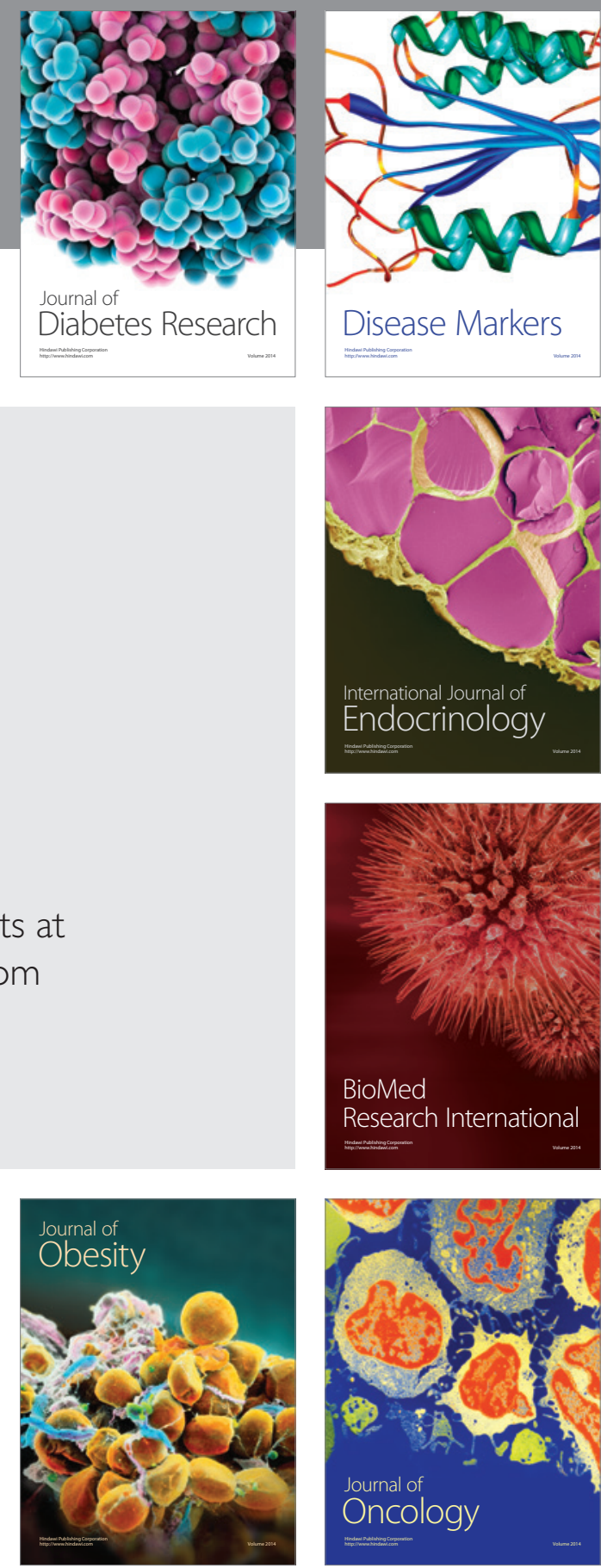

Disease Markers
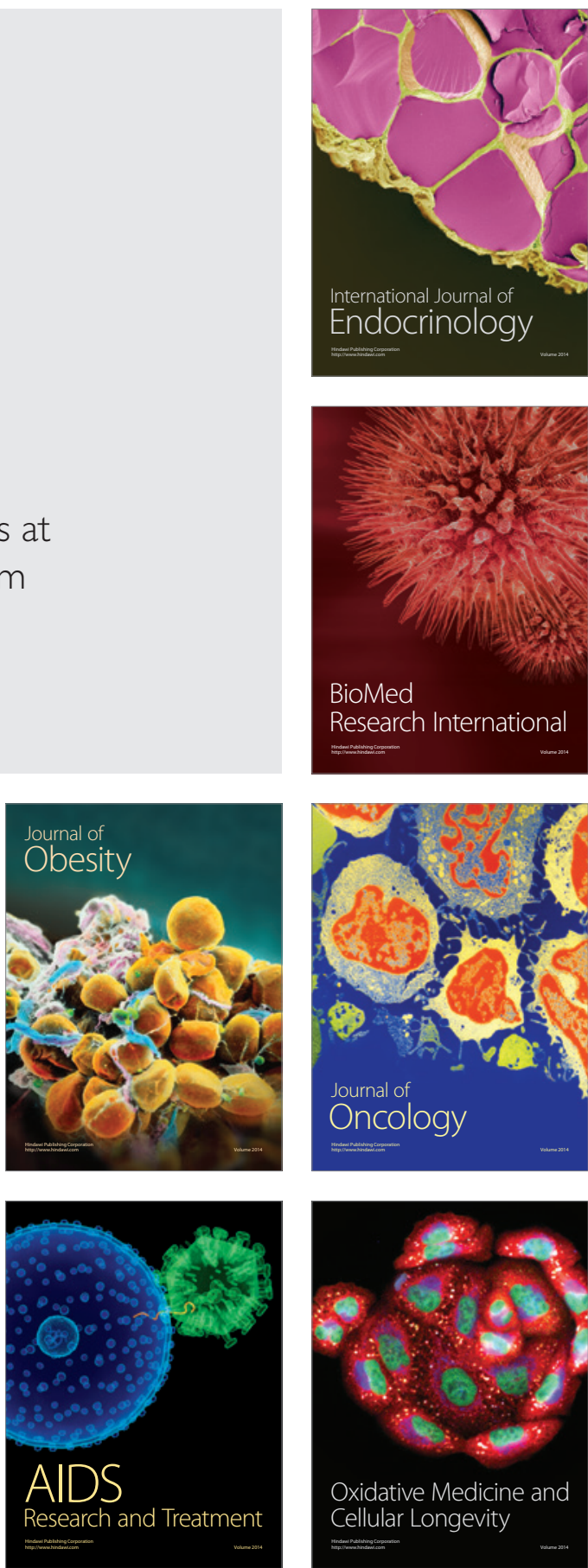